\title{
The impact of disease progression on perceived health status and quality of life of long-term cancer survivors
}

\author{
Melissa S. Y. Thong • Floortje Mols • \\ Jan-Willem W. Coebergh • Jan A. Roukema • \\ Lonneke V. van de Poll-Franse
}

Received: 23 March 2009 / Accepted: 8 June 2009 / Published online: 26 June 2009

(C) The Author(s) 2009. This article is published with open access at Springerlink.com

\begin{abstract}
Introduction The number of cancer survivors experiencing disease progression (DP) is increasing with the number of cancer survivors. However, little is known whether DP affects health-related quality of life (HRQL) of long-term cancer survivors. We aimed therefore to compare the health status (HS) and HRQL of DP and disease-free (DF) survivors up to 15 years after initial diagnosis.

Methods 232 cancer survivors with DP identified through the Eindhoven Cancer Registry were matched with 232 DF survivors of similar demographic and clinical characteristics. Patients completed generic HS (SF-36) and cancer-specific HRQL (QOL-CS) questionnaires 5-15 years after diagnosis. Results Compared with DF survivors, DP survivors exhibited significantly lower scores on all SF-36 and QOLCS (except spiritual well-being) dimensions. DF survivors had better scores than the normative population on all SF-36 dimensions. Among survivors with DP, those with short
\end{abstract}

M. S. Y. Thong $(\bowtie) \cdot$ F. Mols $\cdot$ J.-W. W. Coebergh

L. V. van de Poll-Franse

Comprehensive Cancer Center South (CCCS),

Eindhoven Cancer Registry,

P.O. Box 231, 5600 AE Eindhoven, The Netherlands

e-mail: M.Thong@ikz.nl

F. Mols $\cdot$ L. V. van de Poll-Franse

CoRPS-Center of Research on Psychology in Somatic Diseases,

Department of Medical Psychology, Tilburg University,

Tilburg, The Netherlands

J.-W. W. Coebergh

Department of Public Health,

University Medical Center Rotterdam,

Rotterdam, The Netherlands

\section{J. A. Roukema}

Department of Surgery, St Elisabeth Hospital,

Tilburg, The Netherlands survival ( $<5$ years) had significantly poorer HS scores on all dimensions except bodily pain compared with the normative population. Comparatively, the long survival ( $\geq 5$ years) DP group had better HRQL than the short DP group but poorer HRQL than the normative population. In multivariate analyses, DP and DF survival time were independently associated with aspects of HS and HRQL in cancer survivors.

Discussions/Conclusions DP cancer survivors have poorer long-term HS and HRQL compared with DF survivors. However, there is suggestion that HS and HRQL does improve over time following DP.

Implication for Cancer Survivors Although DP survivors report poorer long-term HRQL compared with DF cancer survivors, results suggest that time can attenuate the distress of DP on HRQL. Psycho-educational programs could help to increase patients' sense of empowerment and personal control should DP occur.

Keywords Cancer - Disease progression · Health status . Long-term survivors · Quality of life · Recurrence

\section{Introduction}

Due to earlier diagnosis and improvement in cancer treatments, cancer survivors are living longer after an initial diagnosis of cancer [1]. Cancer is now often considered a chronic illness rather than a life-threatening disease [2]. However with longer survival after initial diagnosis, the number of cancer survivors experiencing disease progression (DP) such as a recurrence, a metastasis or a new tumor could also increase [3-5]. Survivors of a first primary cancer have an increased risk of up to $50 \%$ of developing subsequent primary cancers $[6,7]$, while recurrence rates 
range from $5 \%$ to $>30 \%$ for endometrial cancer, prostate cancer, and non-Hodgkin lymphoma [8-10].

Previous studies suggest that DP can cause greater distress than the initial cancer diagnosis [11], and is associated with reduced psychological well-being [12-16], poorer physical functioning [12, 17], high symptom burden [15, 18], and poorer health-related quality of life (HRQL) [19, 20]. However, most of these studies focused on breast cancer, had small sample sizes, had no comparison with a diseasefree control group or had short follow-up since DP. To our knowledge, no study has explored the association between DP, survival time since progression, and HRQL of long-term cancer survivors (i.e. patients who are alive $>5$ years since initial diagnosis).

In this secondary analysis of a cancer survivorship study whose details are reported elsewhere [21], we aim to investigate the effects of DP on health status (HS) and HRQL of long-term survivors of cancer of the endometrium or prostate, or Hodgkin's or non-Hodgkin's lymphoma. In specific, we hypothesized that DP survivors will have poorer HS and HRQL compared with patients who remain diseasefree (DF) after initial diagnosis.

\section{Methods}

Setting and participants

From the Eindhoven Cancer Registry (ECR), all patients diagnosed with either Hodgkin's lymphoma or nonHodgkin's lymphoma between 1989 and 1998, or endometrial or prostate cancer between 1994 and 1998 were eligible for participation in a population-based crosssectional survey. Hodgkin's and non-Hodgkin's lymphoma have a longer inclusion period to increase the sample size. The ECR records data on all patients newly diagnosed with cancer in the southern part of the Netherlands, an area with 2.3 million inhabitants, 10 hospitals with 18 locations and two large radiotherapy institutes [22]. After excluding all persons who had died prior to 1 November 2004 (as according to the Central Bureau for Genealogy which collects information on all deceased Dutch citizens via the civil municipal registries), data collection started in November 2004. A local certified Medical Ethics Committee approved of this study. Additional details of the study methodology have been described elsewhere [21].

\section{Clinical characteristics}

Patients' sociodemographic and clinical information were available from the ECR. The ECR routinely collects data on tumor characteristics, including date of diagnosis, tumor grade according to the Tumor-Node-Metastasis clinical classification [23], clinical stage [23], treatment, and patient background characteristics including date of birth and comorbidity at the time of diagnosis. Comorbidity was categorized according to an adapted Charlson comorbidity index [24]. In this study, cancer was not included as a comorbid condition. Socioeconomic status was determined by an indicator developed by Statistics Netherlands based on individual fiscal data from the year 2000 on the economic value of the home and household income, and provided as aggregate level for each postal code (average 17 households) [25], which were then categorized into tertiles.

DP was defined as at least the first recurrence, metastasis or new primary tumor experienced since initial diagnosis. DP was indicated by patients in the self-report questionnaire. Patients were asked to report on any DP and the month/year that this was diagnosed. Affirmative answers were then confirmed by registry staff against ECR records, together with information on the date of this new diagnosis and subsequent treatment received. For DP patients, survival time was calculated from the date of last DP diagnosis to time of survey. If the date of last diagnosis was not available from ECR records, we used the date as reported by the patient. The survival time for DF patients was calculated from date of initial diagnosis to time of survey. We used the term 'survival time' rather than 'DF time' to indicate time since last diagnosis to time of study although mortality is not an outcome in this study as we cannot ascertain that patients are DF following DP. Patients with DP were matched with patients who remained DF since initial diagnosis till time of survey on: a) type of cancer, b) cancer stage at initial diagnosis, c) tumor grade at initial diagnosis, d) age at initial diagnosis ( \pm 2 years), e) age at time of survey ( \pm 5 years) and $f)$ number of years since initial diagnosis ( \pm 3 years).

\section{Data collection}

Cancer survivors were informed of the study via a letter from their (ex)-attending physician. The letter explained that the completion and return of the enclosed questionnaire indicate patient's consent to participation in the study and to agree to the linkage of the questionnaire data with their disease history in the ECR. Patients were reassured that non-participation had no consequences on their follow-up care or treatment. Non-respondents were sent a reminder letter within 2 months.

\section{Measures}

Generic health status was assessed with the Dutch version of the SF-36 questionnaire [26]. The SF-36 has 36 items measuring eight dimensions of health status: physical 
functioning, role limitations due to physical problems, role limitations due to emotional problems, social functioning, mental health, vitality, bodily pain, and general health perceptions. Items in each dimension are added together to form subscale scores, which are transformed to a 0-100 scale, with higher scores indicating better perceived health. The eight subscale scores can be further combined into the physical (PCS) and mental (MCS) component summary score. For the SF-36, differences of $\geq 5$ points in the general health domain [27], 6.5 points in the physical domain and 7.9 points in the mental health domain were considered clinically meaningful [28]. We determined clinically meaningful differences for other subscales with Norman's 'rule of thumb', whereby an $\approx 0.5 \mathrm{SD}$ difference indicates a threshold of discriminant change in HRQL scores of a chronic illness [29]. The internal consistency and reliability of all scales had Cronbach alpha values above 0.70 as recommended for group comparisons.

The validated Dutch version of the Quality of LifeCancer Survivors (QOL-CS) questionnaire assessed HRQL issues specific to cancer survivors [30]. The QOL-CS includes 45 visual analogue scales, with each ranging from 0 (worst outcome) to 10 (best outcome). These 45 visual analogue scales are grouped into four multi-item subscales on well-being: physical, psychological, social, and spiritual. Similarly, Norman's rule was used to indicate clinically significant differences [29].

\section{Statistical analyses}

All statistical analyses were performed using SAS (version 9.1 for Windows, SAS institute Inc., Cary NC). Differences between demographic and clinical characteristics, and HS and HRQL scores between the two groups were compared with chi-square test or t-test where appropriate. Nonparametric equivalents were applied when normality and homogeneity assumptions were violated. The association between disease progression and long-term HRQL was investigated with linear regression analyses, with adjustments for DF survival time, marital status, SES, and comorbidity. Statistical differences were indicated if $p<$ 0.05 and reported $p$-values were two-sided.

\section{Results}

Of the eligible survivors, 1511 (80\%) returned a completed questionnaire (Fig. 1). Details of the data collection process for the whole sample, and the baseline characteristics of respondents and non-respondents have been discussed elsewhere [21]. From the respondents, 232 DP survivors (13 Hodgkin's lymphoma, 53 non-Hodgkin's lymphoma,
19 endometrial cancer, and 147 prostate cancer) were matched with 232 DF survivors of similar clinical and demographic characteristics (Table 1). No differences on the relevant clinical and demographic characteristics indicated a successful match of the two groups. Comparison on other demographic and clinical variables suggested that DP survivors had more comorbid conditions and were less likely to be working than DF survivors. Among the DP survivors, 30 had a second DP. The time since last progression to the date of completion of questionnaire was, on average, $3.4 \pm 3.3$ years.

Table 2 outlines the HS and HRQL scores of DP and DF survivors. Although statistical significant differences in all dimensions of generic HS between both groups were noted, clinically significant differences were observed in the dimensions of general health, physical function, role function physical, role function emotional, and the physical component score. On the cancer-specific QOL-CS, DP survivors had statistically poorer scores on physical, psychological, social, and total well-being, but not spiritual well-being. However, only the differences in physical, social, and total well-being scores showed clinical significance. Similar HS and HRQL results were found between both the DP and DF groups when analysis was limited to either prostate cancer survivors or survivors of endometrial cancer and the lymphomas (excluding prostate cancer) (data not shown).

The generic HS of DF and DP survivors was compared with that of a normative population, standardized for age. DF survivors had better HS than the normative population on all dimensions of the SF-36, with physical functioning, bodily pain, social functioning, role functioning emotional, and physical component summary score statistically significantly better than that of the normative population (Fig. 2). Among the DP group with known time of progression, survival time was dichotomized into short $(<5$ years) and long ( $\geq 5$ years) survival since progression. Compared with the normative population, the short DP group had statistically significantly poorer HS scores on all dimensions except on bodily pain. Clinically significant differences between the two groups were noted in general health and mental component summary scores. Similarly, the long DP group had poorer HS than the normative population, although only general health and the mental component summary scores showed statistically but not clinically significant differences. However long DP survivors report having better HS than short DP survivors (Table 3). Long DP survivors exhibited significantly better mental component summary score and the difference in general health scores approached significance. For those DP patients $(n=$ 29) whose date of DP were not known, their HS scores were generally compatible with those of the long DP survivors (data not shown). 
FIGURE 1 Flow-chart of the data collection process.

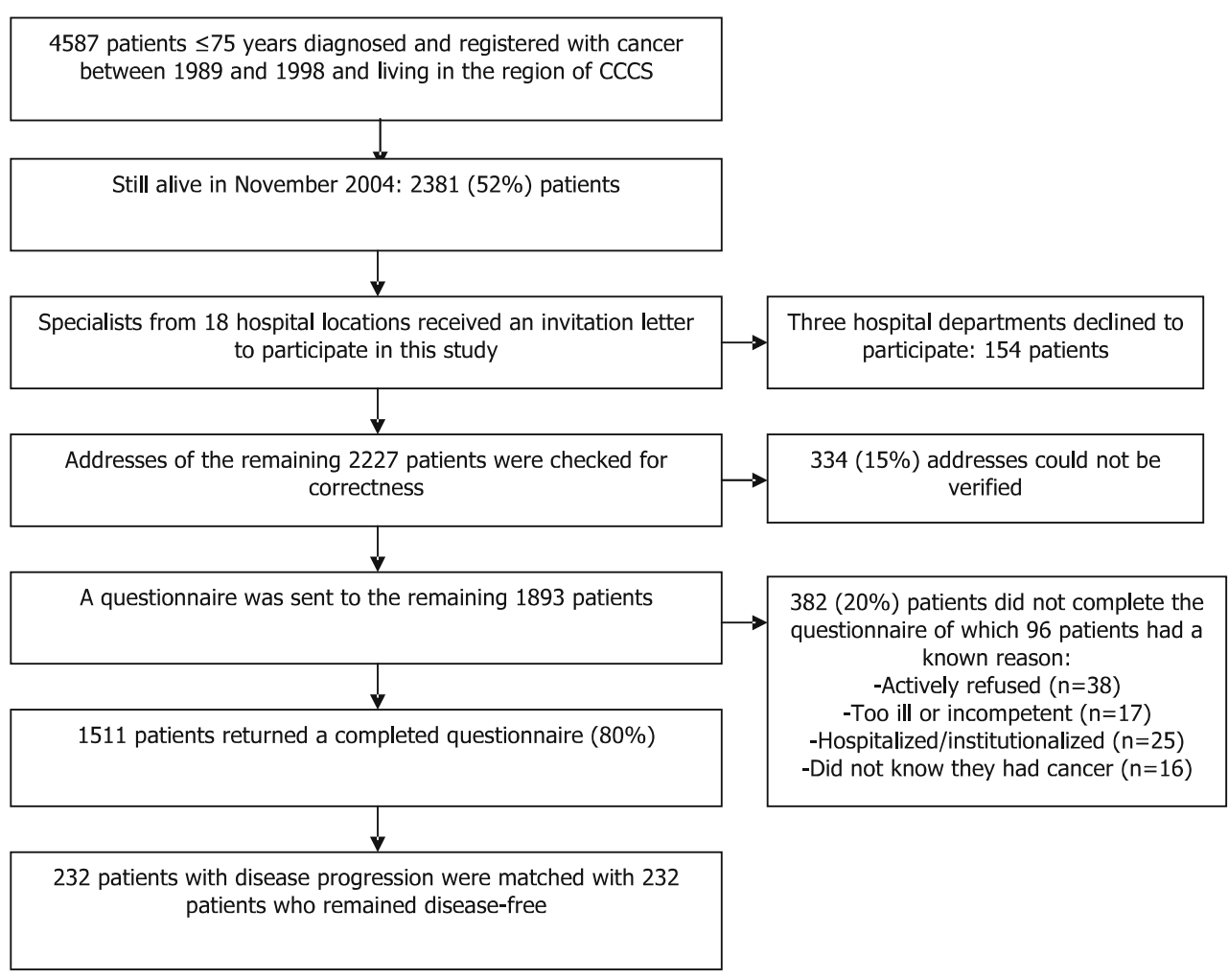

In univariate analyses, DP independently predicted HS and HRQL except for spiritual well-being (data not shown). Similarly, DF time was associated with most dimensions of the SF-36 and QOL-CS except for bodily pain and spiritual well-being (data not shown). In multivariate analyses, DP remained a significant predictor on most dimensions of the SF-36 and QOL-CS (Table 4). However, the association of DF time with HS and HRQL was reduced in the multivariate model, remaining significant only in the dimensions of role functioning emotional, mental health, mental component summary score, and total well-being. Poorer scores on all domains of the SF-36 and QOL-CS (except for spiritual well-being) were independently predicted by comorbidity in both univariate and multivariate analyses.

\section{Discussion}

As hypothesized, our study showed that DP cancer survivors had poorer HS and HRQL than DF cancer survivors of similar clinical manifestation and age. Patients with DP within 5 years of our study reported the lowest HS and HRQL. Cancer survivors who remain DF up to 10 years since initial diagnosis have HS and HRQL better or comparable to an age-matched normative population. Moreover, DP and comorbid conditions predicted that aspects of long-term HS and HRQL would be lower.
Compared with DF survivors, DP survivors had clinically significant poorer scores on several aspects of HS and HRQL, namely that of physical, social, and psychological functioning. Similarly in a matched study, Oh, et al reported that survivors with recurrent breast cancer had poorer HRQL on multiple domains compared with DF survivors [19]. Survivors of multiple cancers had also poorer global HRQL, lower vitality, more cancer-specific stress and lower existential well-being compared with survivors of single primary cancer [31]. In another study, breast cancer survivors with and without recurrence had similar HRQL scores at baseline. At 8-years follow-up, although the HRQL of disease-free survivors had improved significantly, the HRQL scores of those with a recurrence remained similar to their baseline assessment [13].

Higher prevalence of comorbid conditions among DP survivors could partly explain their perceived poorer physical and social aspects of HS and HRQL. Moreover, comorbidity was a significant predictor of HS and HRQL in our multivariate analysis. Similarly in a study using CaPSURE data, prostate cancer survivors with moderate to severe cardiovascular comorbidity had significantly poorer physical component scale scores over 24 months follow-up compared with patients with no comorbidity [32]. A study of cervical cancer survivors also reported significant association between comorbidity and HRQL [33].

Although we performed a cross-sectional study, our results suggest that long-term DP survivors do report better 
Table 1 Demographic and clinical characteristics of patients by disease status

\begin{tabular}{|c|c|c|c|}
\hline & No progression $(n=232)$ & Disease progression $(n=232)$ & p-value \\
\hline \multicolumn{4}{|l|}{ Types of cancer $(\%)$} \\
\hline Hodgkin's lymphoma & $13(6)$ & $13(6)$ & \\
\hline Non-Hodgkin's lymphoma & $53(23)$ & $53(23)$ & \\
\hline Endometrial cancer & $19(8)$ & $19(8)$ & \\
\hline Prostate cancer & $147(63)$ & $147(63)$ & \\
\hline Mean age at time of survey & $69.0 \pm 10.4$ & $68.8 \pm 10.3$ & 0.40 \\
\hline \multicolumn{4}{|l|}{ Age at time of survey $(\%)$} \\
\hline$<55$ & $23(10)$ & $21(9)$ & \\
\hline $55-64$ & $39(17)$ & $41(18)$ & \\
\hline $65-74$ & $94(40)$ & $97(42)$ & 0.87 \\
\hline $75-84$ & $73(31)$ & $72(31)$ & \\
\hline$>85$ & $3(1)$ & $1(0.4)$ & \\
\hline Mean years since diagnosis of initial cancer & $8.2 \pm 1.7$ & $8.2 \pm 1.8$ & 0.72 \\
\hline \multicolumn{4}{|l|}{ Marital status (\%) } \\
\hline Married & $169(76)$ & $178(77)$ & \\
\hline Single/Divorced & $26(12)$ & $26(11)$ & 0.97 \\
\hline Widowed & $26(12)$ & $26(11)$ & \\
\hline \multicolumn{4}{|l|}{ Educational level $^{\mathrm{a}}(\%)$} \\
\hline Low & $108(49)$ & $98(44)$ & \\
\hline Medium & $66(30)$ & $87(39)$ & 0.14 \\
\hline High & $46(21)$ & $36(17)$ & \\
\hline \multicolumn{4}{|l|}{ Employment status (\%) } \\
\hline Not working/ retired & $185(84)$ & $201(88)$ & 0.03 \\
\hline Working & $35(16)$ & $28(12)$ & \\
\hline \multicolumn{4}{|l|}{ Socioeconomic status (\%) } \\
\hline Low & $49(21)$ & $51(22)$ & \\
\hline Medium & $97(42)$ & $92(40)$ & 0.97 \\
\hline High & 77 (33) & 79 (34) & \\
\hline \multicolumn{4}{|l|}{ Stage (\%) } \\
\hline 1 & $74(32)$ & $75(32)$ & \\
\hline 2 & $107(46)$ & $106(46)$ & 0.99 \\
\hline 3 & $16(7)$ & $15(6)$ & \\
\hline 4 & $35(15)$ & $36(16)$ & \\
\hline \multicolumn{4}{|l|}{ Tumor Grade ${ }^{\mathrm{b}}(\%)$} \\
\hline Good & $55(24)$ & $53(22)$ & \\
\hline Moderate & $76(33)$ & $76(33)$ & \\
\hline Poor & $35(15)$ & $36(16)$ & 0.97 \\
\hline Undifferentiated & 0 & $1(0.4)$ & \\
\hline T-cell (lymphoma) & $2(1)$ & $3(1)$ & \\
\hline B-cell (lymphoma) & $51(22)$ & $50(21)$ & \\
\hline \multicolumn{4}{|l|}{ Comorbidity (\%) } \\
\hline None & $91(39)$ & $75(32)$ & \\
\hline 1 & $90(39)$ & $82(35)$ & 0.04 \\
\hline$>1$ & $51(22)$ & $75(32)$ & \\
\hline \multicolumn{4}{|l|}{ Most common comorbid conditions (\%) } \\
\hline Hypertension & $60(26)$ & $62(27)$ & 0.83 \\
\hline Arthritis & $51(22)$ & $61(26)$ & 0.28 \\
\hline Diabetes mellitus & $23(10)$ & $32(14)$ & 0.19 \\
\hline Asthma & $27(12)$ & $29(13)$ & 0.77 \\
\hline
\end{tabular}


Table 1 (continued)

\begin{tabular}{|c|c|c|c|}
\hline & No progression $(n=232)$ & Disease progression $(n=232)$ & p-value \\
\hline Mean years since disease progression & & $3.4 \pm 3.3$ & \\
\hline \multicolumn{4}{|l|}{ Years since disease progression ${ }^{\mathrm{c}}(\%)$} \\
\hline$<2$ years & & $94(40)$ & \\
\hline $2-5$ years & & $58(24)$ & \\
\hline$>5$ years & & $51(21)$ & \\
\hline
\end{tabular}

${ }^{a}$ Education: Low (no or primary school); Medium (lower general secondary education or vocational training); High (pre-university education, high vocational training, university)

b 13 patients with Hodgkin's lymphoma were matched excluding the criterion tumor grade as these were not available

${ }^{\mathrm{c}} 29$ patients have unknown date of progression

HS and HRQL scores over time. DP patients with shorter survival time since last diagnosis had significantly poorer HS and HRQL compared with an age-standardized normative population. However, we noted that DP survivors with a progression $\geq 5$ years ago had higher HS and HRQL scores than those with shorter survival time since last diagnosis. That we only found a significant difference in the mental component summary score between these two groups could be due to lack of power from the small sample of long DP survivors. A longitudinal study showed that patients with recurrent cancer had poorer HRQL compared with single diagnosis patients at 12 months follow-up, although the HRQL among the group with recurrence showed improvement over time [17]. Moreover, in that study while patients in both groups reported improved psychological well-being over time, patients with a recurrence had better psychological well-being at baseline and follow-up than patients with a single diagnosis. Similarly, a Swedish longitudinal study with recurrent breast cancer survivors reported a significant decrease in distress at 6 months follow-up [34]. This suggests that cancer survivors might initially be distressed with the diagnosis of DP $[11,13]$ but do have the resilience to adapt and cope with the new diagnosis and subsequent treatment over time. Gotay, et al found that survivors of multiple primary cancers coped better with the second diagnosis than the first [31]. Together with our current results, these studies suggest that previous experiences with cancer and its treatment could buffer a patient from the psychological distress of coping with DP and subsequent treatment. This reconceptualization of HRQL over the course of the disease trajectory has been termed 'response shift', and refers to the changes in self-evaluation of HRQL as cancer survivors adapt to their disease and treatment [35]. We further
Table 2 Mean scores $( \pm \mathrm{SD})$ of SF-36 and QOL-CS by disease status

PCS Physical Component

Summary score, MCS Mental

Component Summary score,

QOL-CS Quality of Life-Cancer

Survivors

${ }^{a}$ indicates that the difference in the HRQL score is also

clinically significant

\begin{tabular}{lccc}
\hline & Disease progression $(n=232)$ & No progression $(n=232)$ & p-value \\
\hline SF-36 & & & \\
General health & $48.9 \pm 23.5$ & $62.9 \pm 22.1$ & $<0.0001^{\mathrm{a}}$ \\
Physical function & $60.5 \pm 29.7$ & $73.2 \pm 25.0$ & $<0.0001^{\mathrm{a}}$ \\
Role function-physical & $48.0 \pm 45.7$ & $70.1 \pm 40.1$ & $<0.0001^{\mathrm{a}}$ \\
Bodily pain & $69.8 \pm 27.0$ & $78.2 \pm 22.9$ & $<0.001$ \\
Vitality & $57.0 \pm 24.3$ & $67.5 \pm 20.8$ & $<0.0001$ \\
Social functioning & $72.6 \pm 26.9$ & $83.5 \pm 21.4$ & $<0.0001$ \\
Role function-emotional & $64.6 \pm 44.1$ & $86.5 \pm 28.7$ & $<0.0001^{\mathrm{a}}$ \\
Mental health & $70.8 \pm 20.7$ & $77.5 \pm 16.8$ & $<0.001$ \\
PCS & $40.0 \pm 11.7$ & $46.0 \pm 9.8$ & $<0.0001^{\mathrm{a}}$ \\
MCS & $49.0 \pm 11.2$ & $51.0 \pm 8.7$ & $<0.0001$ \\
QOL-CS & & & $<0.0001^{\mathrm{a}}$ \\
Physical well-being & $7.2 \pm 1.9$ & $8.0 \pm 1.8$ & $<0.0001$ \\
Psychological well-being & $5.9 \pm 1.8$ & $6.9 \pm 1.6$ & $<0.0001^{\mathrm{a}}$ \\
Social well-being & $6.6 \pm 1.7$ & $7.2 \pm 1.7$ & $\mathrm{n} . \mathrm{s}$. \\
Spiritual well-being & $4.6 \pm 1.7$ & $4.8 \pm 1.4$ & $<0.0001^{\mathrm{a}}$ \\
Total well-being & $6.1 \pm 1.4$ & $6.8 \pm 1.3$ & \\
\hline
\end{tabular}




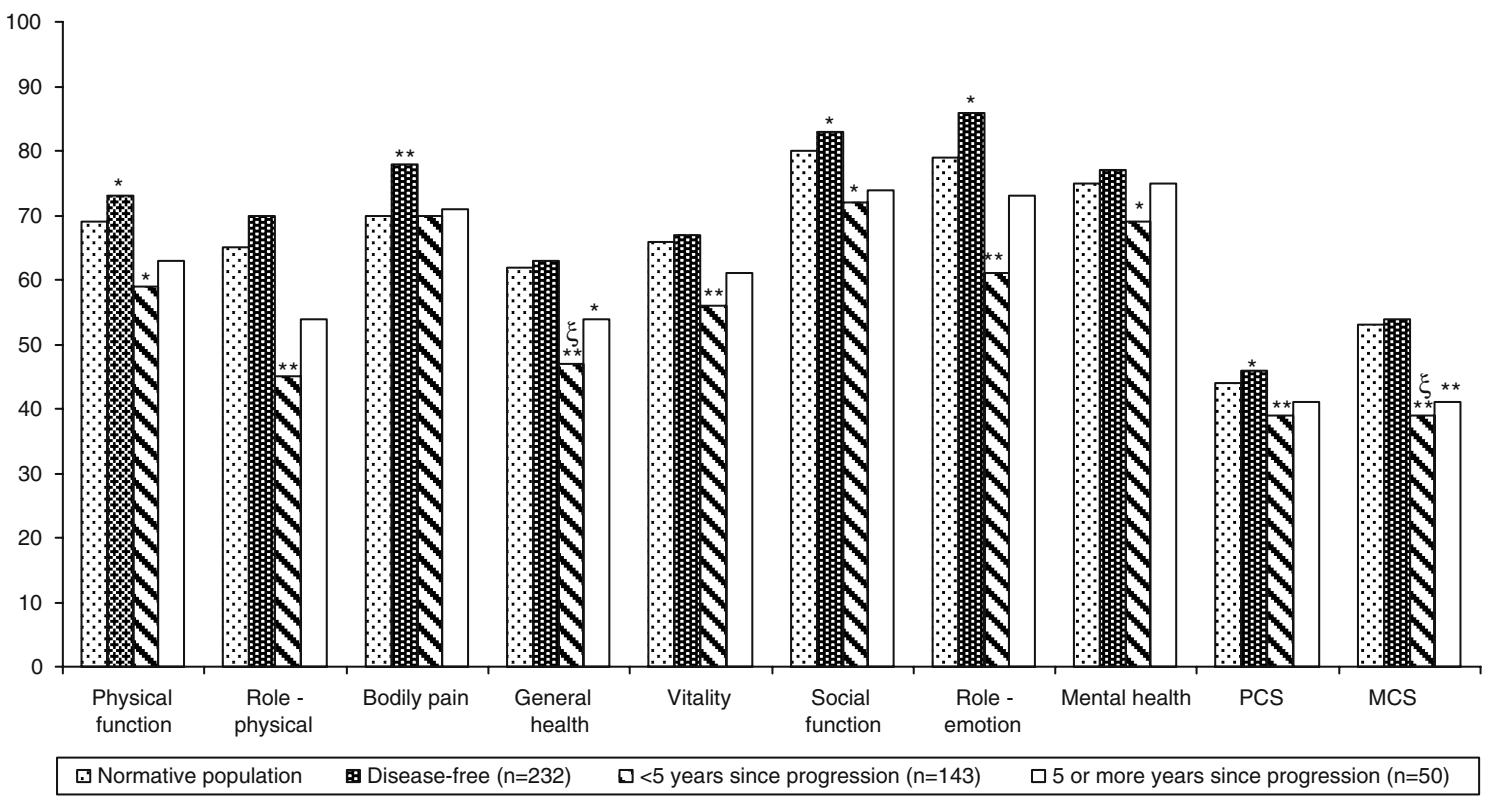

FIGURE 2 Comparison of SF-36 scores according to disease status to the normative population standardized for age. An asterisk above a subgroup indicates a difference in the mean score between that subgroup and the normative population. PCS=Physical Component
Summary score. MCS=Mental Component Summary score. ${ }^{* *} p<$ $0.001 ; * p<0.05$. $\xi$ Indicates clinically significant difference in mean score between the subgroup and the norm population.

a normative population. Having survived cancer and remaining DF for up to 15 years since initial diagnosis could improve survivors' self-evaluation of HRQL. DF survivors finding benefit following their cancer diagnosis and its subsequent cure could also impact on their HRQL. In a study of 96 breast cancer survivors of whom the majority remained DF, baseline benefit finding predicted
Table 3 Mean scores ( \pm SD) of SF-36 and QOL-CS of patients with disease progression by survival time since progression ${ }^{\mathrm{a}}$

PCS Physical Component Summary Score, MCS Mental Component Summary Score, QOL-CS Quality of Life-Cancer Survivors

${ }^{a}$ time since progression is missing for 29 patients

\begin{tabular}{lccc}
\hline & $<5$ years $(n=143)$ & $\geq 5$ years $(n=50)$ & $p$-value \\
\hline SF-36 & & & \\
General health & $47.1 \pm 23.8$ & $54.2 \pm 23.8$ & 0.05 \\
Physical function & $59.2 \pm 30.6$ & $63.2 \pm 28.9$ & 0.51 \\
Role function-physical & $45.1 \pm 45.7$ & $53.8 \pm 45.3$ & 0.27 \\
Bodily pain & $69.9 \pm 26.2$ & $70.7 \pm 29.5$ & 0.66 \\
Vitality & $56.2 \pm 23.5$ & $60.8 \pm 26.6$ & 0.20 \\
Social functioning & $72.0 \pm 26.2$ & $74.3 \pm 32.1$ & 0.20 \\
Role function-emotional & $61.1 \pm 44.9$ & $72.6 \pm 40.4$ & 0.16 \\
Mental health & $69.4 \pm 21.3$ & $74.5 \pm 20.0$ & 0.10 \\
PCS & $39.6 \pm 11.5$ & $41.1 \pm 12.7$ & 0.48 \\
MCS & $47.7 \pm 11.2$ & $51.5 \pm 11.1$ & 0.03 \\
QOL-CS & & & \\
Physical well-being & $7.1 \pm 2.0$ & $7.5 \pm 1.8$ & 0.17 \\
Psychological well-being & $5.8 \pm 1.9$ & $6.1 \pm 1.6$ & 0.45 \\
Social well-being & $6.5 \pm 1.7$ & $6.8 \pm 1.5$ & 0.35 \\
Spiritual well-being & $4.5 \pm 1.5$ & $4.9 \pm 1.7$ & 0.13 \\
Total well-being & $6.0 \pm 1.4$ & $6.3 \pm 1.3$ & 0.19 \\
\hline
\end{tabular}




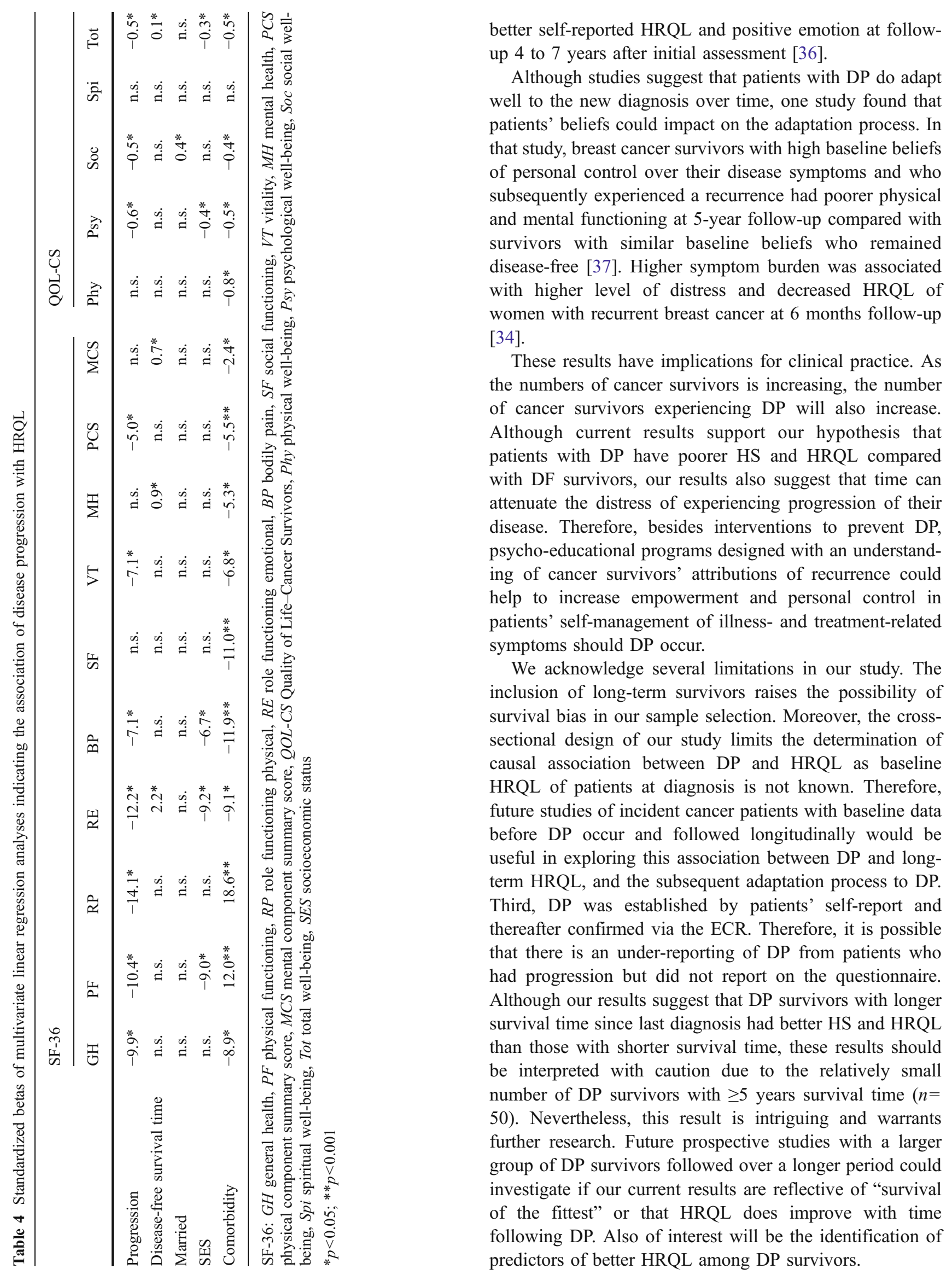


Strong points of our study include the HS and HRQL assessments of patients with DP up to 15 years since initial cancer diagnosis. Moreover, inclusion of the length of time since DP for consideration when assessing long-term HS and HRQL in cancer survivors has, to our knowledge, not been reported previously.

\section{Conclusions}

Cancer survivors who experience DP report poorer longterm HS and HRQL compared with disease-free survivors. However, results suggest that DP survivors do report better HS and HRQL over time.

Financial disclosure/Conflict of interest None declared from all authors

Open Access This article is distributed under the terms of the Creative Commons Attribution Noncommercial License which permits any noncommercial use, distribution, and reproduction in any medium, provided the original author(s) and source are credited.

\section{References}

1. Karim-Kos HE, de Vries E, Soerjomataram I, Lemmens V, Siesling S, Coebergh JW. Recent trends of cancer in Europe: a combined approach of incidence, survival and mortality for 17 cancer sites since the 1990s. Eur J Cancer. 2008;44:1345-89.

2. Ganz PA. Why and how to study the fate of cancer survivors: observations from the clinic and the research laboratory. Eur $\mathrm{J}$ Cancer. 2003;39:2136-41.

3. Soerjomataram I, Louwman MW, Ribot JG, Roukema JA, Coebergh JW. An overview of prognostic factors for long-term survivors of breast cancer. Breast Cancer Res Treat. 2008;107:309-30.

4. Tran W, Serio AM, Raj GV, Dalbagni G, Vickers AJ, Bochner $\mathrm{BH}$, et al. Longitudinal risk of upper tract recurrence following radical cystectomy for urothelial cancer and the potential implications for long-term surveillance. J Urol. 2008;179:96-100.

5. Soerjomataram I, Louwman WJ, de Vries E, Lemmens VE, Klokman WJ, Coebergh JW. Primary malignancy after primary female breast cancer in the South of the Netherlands, 1972-2001. Breast Cancer Res Treat. 2005;93:91-5.

6. Soerjomataram I, Louwman WJ, Lemmens VE, de Vries E, Klokman WJ, Coebergh JW. Risks of second primary breast and urogenital cancer following female breast cancer in the south of The Netherlands, 1972-2001. Eur J Cancer. 2005;41:2331-7.

7. Travis LB. The epidemiology of second primary cancers. Cancer Epidemiol Biomarkers Prev. 2006;15:2020-6.

8. Scholten AN, van Putten WL, Beerman H, Smit VT, Koper PC, Lybeert ML, et al. Postoperative radiotherapy for Stage 1 endometrial carcinoma: long-term outcome of the randomized PORTEC trial with central pathology review. Int J Radiat Oncol Biol Phys. 2005;63:834-8.

9. Secin FP, Bianco FJ Jr, Vickers AJ, Reuter V, Wheeler T, Fearn $\mathrm{PA}$, et al. Cancer-specific survival and predictors of prostatespecific antigen recurrence and survival in patients with seminal vesicle invasion after radical prostatectomy. Cancer 2006;106: 2369-75.

10. Tilly H, Dreyling M. Diffuse large B-cell non-Hodgkin's lymphoma: ESMO clinical recommendations for diagnosis, treatment and follow-up. Ann Oncol. 2008;19(Suppl 2):ii67-9.

11. Cella DF, Mahon SM, Donovan MI. Cancer recurrence as a traumatic event. Behav Med. 1990;16:15-22.

12. Silberfarb PM, Maurer LH, Crouthamel CS. Psychosocial aspects of neoplastic disease: I. Functional status of breast cancer patients during different treatment regimens. Am J Psychiatry. 1980;137:450-5.

13. Andersen BL, Shapiro CL, Farrar WB, Crespin T, WellsDigregorio S. Psychological responses to cancer recurrence. Cancer 2005;104:1540-7.

14. Lee MK, Son BH, Hwang SY, Han W, Yang JH, Lee S, et al. Factors affecting health-related quality of life in women with recurrent breast cancer in Korea. Qual Life Res. 2007;16:559-69.

15. Kenne Sarenmalm E, Ohlen J, Jonsson T, Gaston-Johansson F. Coping with recurrent breast cancer: predictors of distressing symptoms and health-related quality of life. J Pain Symptom Manage. 2007;34:24-39.

16. Okamura M, Yamawaki S, Akechi T, Taniguchi K, Uchitomi Y. Psychiatric disorders following first breast cancer recurrence: prevalence, associated factors and relationship to quality of life. Jpn J Clin Oncol. 2005;35:302-9.

17. Yang HC, Thornton LM, Shapiro CL, Andersen BL. Surviving recurrence: psychological and quality-of-life recovery. Cancer 2008;112:1178-87.

18. Camilleri-Brennan J, Steele RJ. The impact of recurrent rectal cancer on quality of life. Eur J Surg Oncol. 2001;27:349-53.

19. Oh S, Heflin L, Meyerowitz BE, Desmond KA, Rowland JH, Ganz PA. Quality of life of breast cancer survivors after a recurrence: a follow-up study. Breast Cancer Res Treat. 2004;87:45-57.

20. Northouse LL, Mood D, Kershaw T, Schafenacker A, Mellon S, Walker J, et al. Quality of life of women with recurrent breast cancer and their family members. J Clin Oncol. 2002;20:4050-64.

21. Mols F, Helfenrath KA, Vingerhoets AJ, Coebergh JW, van de Poll-Franse LV. Increased health care utilization among long-term cancer survivors compared to the average Dutch population: a population-based study. Int J Cancer. 2007;121:871-7.

22. Janssen-Heijnen MLG, Louwman WJ, Van de Poll-Franse LV, Coebergh JWW. Results of 50 years cancer registry in the South of the Netherlands: 1955-2004 (in Dutch). Eindhoven: Eindhoven Cancer Registry; 2005.

23. UICC: TNM Atlas Illustrated Guide to the TNM/pTNM Classification of Malignant Tumors. In: 4th ed., 2nd Revision ed. Berlin: Springer-Verlag; 1992. p. 141-144.

24. Charlson ME, Pompei P, Ales KL, MacKenzie CR. A new method of classifying prognostic comorbidity in longitudinal studies: development and validation. J Chronic Dis. 1987;40:373-383.

25. van Duijn C, Keij I. Sociaal-economische status indicator op postcode niveau. Maandstatistiek van de bevolking. 2002;50:32-5.

26. Aaronson NK, Muller M, Cohen PD, Essink-Bot ML, Fekkes M, Sanderman R, et al. Translation, validation, and norming of the Dutch language version of the SF-36 Health Survey in community and chronic disease populations. J Clin Epidemiol. 1998;51:1055-68.

27. Ware J Jr. SF-36 health survey: manual and interpretation guide. Boston, MA: The Health Institute, New England Medical Center; 1993.

28. Norman GR, Sridhar FG, Guyatt GH, Walter SD. Relation of distribution- and anchor-based approaches in interpretation of changes in health-related quality of life. Med Care. 2001;39:1039-47.

29. Norman GR, Sloan JA, Wyrwich KW. Interpretation of changes in health-related quality of life: the remarkable universality of half a standard deviation. Med Care. 2003;41:582-92.

30. van Dis FW, Mols F, Vingerhoets AJ, Ferrell B, van de PollFranse LV. A validation study of the Dutch version of the Quality 
of Life-Cancer Survivor (QOL-CS) questionnaire in a group of prostate cancer survivors. Qual Life Res. 2006;15:160712 .

31. Gotay CC, Ransom S, Pagano IS. Quality of life in survivors of multiple primary cancers compared with cancer survivor controls. Cancer 2007;110:2101-9.

32. van de Poll-Franse LV, Sadetsky N, Kwan L, Litwin MS. Severity of cardiovascular disease and health-related quality of life in men with prostate cancer: a longitudinal analysis from CaPSURE. Qual Life Res. 2008;17:845-55.

33. Ashing-Giwa KT, Tejero JS, Kim J, Padilla GV, Kagawa-Singer M, Tucker MB, et al. Cervical cancer survivorship in a population based sample. Gynecol Oncol. 2009;112:358-64.
34. Kenne Sarenmalm E, Ohlen J, Oden A, Gaston-Johansson F. Experience and predictors of symptoms, distress and healthrelated quality of life over time in postmenopausal women with recurrent breast cancer. Psychooncology 2008;17:497-505.

35. Sprangers MA, Schwartz CE. Integrating response shift into health-related quality of life research: a theoretical model. Soc Sci Med. 1999;48:1507-15.

36. Carver CS, Antoni MH. Finding benefit in breast cancer during the year after diagnosis predicts better adjustment 5 to 8 years after diagnosis. Health Psychol. 2004;23:595-8.

37. Tomich PL, Helgeson VS. Cognitive adaptation theory and breast cancer recurrence: are there limits? J Consult Clin Psychol. 2006;74:980-7. 